Ambiente \& Água - An Interdisciplinary Journal of Applied Science
ISSN 1980-993X - doi:10.4136/1980-993X
www.ambi-agua.net
E-mail: ambi-agua@agro.unitau.br

\title{
Pesquisa de fatores de virulência em Pseudomonas aeruginosa isoladas de águas minerais naturais
}

\author{
doi: $10.4136 / a m b i-a g u a .1359$
}

Received: 07 Apr. 2014; Accepted: 24 May 2014

\begin{abstract}
Aline Pereira Pedrosa*; Marcelo Luiz Lima Brandão; Valéria de Mello Medeiros;
Carla de Oliveira Rosas; Silvia Maria Lopes Bricio; Antônio Eugênio Castro Cardoso Almeida
\end{abstract}

\author{
Fundação Oswaldo Cruz, (FIOCruz), Rio de Janeiro, RJ, Brasil \\ Instituto Nacional de Controle de Qualidade em Saúde, \\ Departamento de Microbiologia \\ *Autor correspondente: e-mail: alinepp.nut@gmail.com, \\ marcelollb_8@hotmail.com, valeria.medeiros@incqs.fiocruz.br, \\ carla.rosas@incqs.fiocruz.br, silvia.bricio@incqs.fiocruz.br, \\ eugenio.almeida@incqs.fiocruz.br
}

\section{RESUMO}

O objetivo deste estudo foi avaliar a formação de biofilme e o perfil de susceptibilidade a antimicrobianos de Pseudomonas aeruginosa isoladas na avaliação da qualidade microbiológica de 80 amostras de águas minerais naturais comercializadas em garrafões de 20 L. Foi realizada a quantificação de $P$. aeruginosa e enterococos; a pesquisa de coliformes totais, coliformes termotolerantes e de clostrídios sulfito redutores (CSR). A produção de biofilme de $P$. aeruginosa foi avaliada em caldo infusão cérebro-coração (BHI) e em água mineral natural estéril nas temperaturas de 25 e $35^{\circ} \mathrm{C}$ por 24 e $48 \mathrm{~h}$. A avaliação da susceptibilidade a antimicrobianos foi realizada pelo teste de difusão em ágar (Kirby-Bauer). De 80 amostras analisadas, $40(50 \%)$ apresentaram qualidade microbiológica insatisfatória segundo a RDC $\mathrm{n}^{\circ} 275 / 05$. Trinta e oito $(47,5 \%)$ amostras apresentaram $P$. aeruginosa, nove $(11,2 \%)$ coliformes totais, quatro $(5,0 \%)$ CSR e uma $(1,2 \%)$ coliformes termotolerantes. Nenhuma amostra apresentou contaminação por enterococos. Dezesseis cepas $(51,6 \%)$ de $P$. aeruginosa foram classificadas como não aderentes ou fracamente aderentes, tanto no BHI quanto na água mineral. Contudo, cinco cepas $(16,1 \%)$ apresentaram-se fortemente aderentes nas duas matrizes, principalmente no caldo $\mathrm{BHI}$ e na temperatura de $25^{\circ} \mathrm{C}$. Cepas resistentes ou com resistência intermediária a antibióticos da classe dos aminoglicosídeos e/ou $\beta$-lactâmicos foram isoladas neste estudo. Concluiu-se que os isolados de $P$. aeruginosa foram capazes de produzir biofilme nas matrizes estudadas e apresentaram resistência a antimicrobianos. Metade das amostras apresentou qualidade microbiológica insatisfatória, principalmente devido à contaminação por $P$. aeruginosa $(47,5 \%)$.

Palavras-chave: clostrídios sulfito redutores, coliformes, águas engarrafadas, biofilme, antimicrobianos. 


\title{
Assessment of virulence factors of Pseudomonas aeruginosa isolated from natural mineral water
}

\begin{abstract}
This study evaluated biofilm formation and antimicrobial susceptibility of Pseudomonas aeruginosa isolated to evaluate the microbiological quality of 80 natural mineral water samples sold in $20 \mathrm{~L}$ bottles. The quantity of $P$. aeruginosa and enterococci was assessed, including total coliforms, thermotolerant coliforms and sulfite-reducing clostridia (SRC). Biofilm production from $P$. aeruginosa was evaluated in brain heart infusion broth (BHI) and sterile natural mineral water at temperatures of 25 and $35^{\circ} \mathrm{C}$ for 24 and $48 \mathrm{~h}$. Antimicrobial susceptibility tests were performed using the agar diffusion method (Kirby-Bauer). Of the 80 samples analyzed, $40(50 \%)$ presented unsatisfactory microbiological quality according to RDC no. 275/05. Thirty-eight (47.5\%) samples presented $P$. aeruginosa, nine (11.2\%) total coliforms, four $(5.0 \%)$ SRC and one $(1.2 \%)$ thermotolerant coliform. Sixteen P. aeruginosa strains $(51.6 \%)$ were classified as non-adherent or weakly adherent, both in BHI as in mineral water. However, five strains $(16.1 \%)$ were strongly adherent in the two matrices, mainly in $\mathrm{BHI}$ at the temperature of $25^{\circ} \mathrm{C}$. The study also isolated resistant or intermediate resistant strains to antibiotics of aminoglycosides and/or $\beta$-lactams classes. It was concluded that $P$. aeruginosa isolates were able to produce biofilm in the studied matrices and presented resistance to antimicrobials. Half of the samples presented unsatisfactory microbiological quality, mostly due to $P$. aeruginosa contamination.
\end{abstract}

Keywords: sulfite-reducing clostridia, coliforms, bottled water, biofilm, antimicrobials.

\section{INTRODUÇÃO}

A preocupação com a qualidade da água, decorrente da progressiva poluição hídrica, é um dos motivos que levam grande parte da população mundial ao consumo de água proveniente de fontes naturais (Leclerc e Moreau, 2002). A água mineral natural é obtida diretamente de fontes naturais ou por extração de águas subterrâneas, sendo caracterizada por um conteúdo definido e constante de determinados sais minerais, oligoelementos e outros constituintes considerando as flutuações naturais (Brasil, 2006). No Brasil, o consumo de águas minerais subiu de 16,6 bilhões de L em 2010 para 17,4 bilhões de L em 2012, sendo o quarto maior mercado consumidor de água engarrafada do mundo (Brasil, 2013a).

A água mineral natural deve apresentar qualidade que garanta ausência de risco à saúde do consumidor, devendo ser captada, processada e envasada obedecendo condições higiênicosanitárias e as boas práticas de fabricação (BPF). No Brasil isto é regulamentado pela RDC n. ${ }^{\circ}$ 173/2006, que dispõe sobre o Regulamento Técnico de Boas Práticas de fabricação para Industrialização e Comercialização de Água mineral Natural e Água Natural (Brasil, 2006).

De acordo com a Organização Mundial de Saúde, mais de 1,8 milhões de pessoas morrem anualmente devido a doenças transmitidas pela água (DTA), tornando as DTA a causa mais importante de morte no mundo (WHO, 2007). No Brasil, entre 2000 e abril de 2013 foram notificados 492 surtos ocasionados por água contaminada. Contudo, os dados disponibilizados não discriminam o tipo de água envolvido em cada surto (Brasil, 2013b).

Os potenciais contaminantes da água mineral incluem uma variedade de espécies saprófitas, tais como aeróbios mesófilos, coliformes, enterococos, Pseudomonas spp. e clostrídios sulfito redutores (CSR) (Kim e Feng, 2001). Dentre estes, P. aeruginosa é a bactéria mais comumente isolada em amostras de águas minerais naturais comercializadas em galões de 20 L no Brasil (Sant'Ana et al., 2003; Brandao et al., 2012). 
P. aeruginosa é um patógeno oportunista que pode causar infecções urinárias, dermatites e uma grande variedade de infecções sistêmicas, principalmente em pacientes imunocomprometidos (Koneman et al., 2001). Esta espécie é um dos patógenos mais relevantes ligados à DTA (Wingender e Flemming, 2011) e é o pseumonídeo mais isolado em casos de infecções hospitalares, sendo uma bactéria de difícil controle devido a sua fácil disseminação pelo ambiente (Koneman et al., 2001).

A contaminação de $P$. aeruginosa em águas minerais naturais pode ocorrer devido à presença natural desta bactéria na flora da água retirada da fonte (Kim e Feng, 2001) ou devido à contaminação durante o processo de envase e engarrafamento, devido à característica de formação de biofilmes nas embalagens reutilizáveis e equipamentos da fábrica (Wingender e Flemming, 2011). Os biofilmes são formados por micro-organismos que crescem de forma agregada, geralmente em superfícies, embebidos em uma matriz de substâncias poliméricas extracelulares (EPS) de sua própria origem que sua constituem a matriz (Yasuhiko et al., 2012).

$P$. aeruginosa consegue se reproduzir em água e outros ambientes com poucos nutrientes (Sant'ana et al., 2003) e possui a capacidade de adquirir resistência a múltiplos antimicrobianos (Koneman et al., 2001). O uso intensivo de antibióticos contribuiu para o aumento da seleção de bactérias resistentes, inclusive em ambientes aquáticos. Desta forma, estas bactérias podem representar um reservatório de resistência, bem como um meio para a propagação e evolução de genes de resistência (Baquero et al., 2008).

O objetivo deste estudo foi avaliar à formação de biofilme e o perfil de susceptibilidade a antimicrobianos em cepas $P$. aeruginosa oriundas da avaliação da qualidade microbiológica de águas minerais naturais comercializadas em garrafões de 20 L no Estado do Rio de Janeiro.

\section{MATERIAL E MÉTODOS}

\subsection{Amostras de água}

Foram analisadas 80 amostras indicativas de água mineral natural, não gaseificada, comercializada em garrafões retornáveis de $20 \mathrm{~L}$, de 33 marcas distintas, codificadas de A até $\mathrm{Z}$ e de A' a G', entre os meses de agosto de 2012 a agosto de 2013, segundo os critérios preconizados pela RDC $\mathrm{n}^{\circ}$ 275/2005 (Brasil, 2005). Foram analisadas 17 amostras da marca "U", seis amostras da marca "E", quatro das marcas "A, M, O e Z", três das marcas "G, J, C' e G'", duas das marcas "F, K, Q, Y, B' e D"' e uma das marcas "B, C, D, H, I, L, N, P, R, S, $\mathrm{T}, \mathrm{V}, \mathrm{W}, \mathrm{X}, \mathrm{A}$ ', E' e F'". As amostras foram adquiridas e levadas ao laboratório e estocadas em temperatura ambiente até o momento da análise.

\subsection{Metodologias de ensaio}

A quantificação de $P$. aeruginosa e enterococos foi realizada pela técnica de tubos múltiplos para determinação do número mais provável (NMP) em $100 \mathrm{~mL}$ (APHA, 2012). Para pesquisa de coliformes totais e termotolerantes foi utilizado o método de presença-ausência (APHA, 2012). A pesquisa de CSR foi realizada pela técnica de enriquecimento em meio líquido (ISO, 1986).

\subsection{Isolamento de Pseudomonas aeruginosa}

Os tubos de caldo acetamida que apresentaram turvação foram semeados, pela técnica de esgotamento, em ágar cetrimide (Merck, Alemanha) e incubados a $35 \pm 2{ }^{\circ} \mathrm{C}$ por $24-\mathrm{h}$. As placas foram observadas sob luz ultravioleta a $365 \mathrm{~nm}$ e foram selecionadas, de cada amostra, uma colônia isolada, verde e com produção de pigmento. As colônias foram semeadas em ágar nutriente (BD, EUA) de forma a colecioná-las para a avaliação da suscetibilidade aos antimicrobianos e formação de biofilme. 


\subsection{Pesquisa de formação de biofilme}

A pesquisa de formação de biofilme dos isolados de $P$. aeruginosa foi realizada segundo a metodologia descrita por Patel e Sharma (2010) com modificações. Foram realizados três experimentos independentes para cada cepa.

As cepas foram semeadas em ágar tripticaseína de soja (TSA) (Merck, Alemanha) e incubadas a $35 \pm 2^{\circ} \mathrm{C}$ por 24-h. Uma colônia isolada foi semeada em dois tubos contendo 5,0 $\mathrm{mL}$ de caldo infusão cérebro-coração (BHI) (Merck, Alemanha) incubados sob agitação de $150 \mathrm{rpm}$ (Orbit-Shaker, Lab-Line, EUA) a $37 \pm 2^{\circ} \mathrm{C}$ por $20-24-\mathrm{h}$. Após a incubação, um tubo de caldo BHI foi utilizado para inoculação de $200 \mu \mathrm{L}$ do crescimento celular, em triplicata, em quatro microplacas de poliestireno contendo 96 orifícios com fundo chato (Microtes ${ }^{\mathrm{TM}}$ Falcon, EUA). O outro tubo contendo a mesma cepa, foi centrifugado a $7500 \mathrm{rpm} / 10 \mathrm{~min}$ (Eppendorf 5804-R, A-4-44, Alemanha), o sobrenadante foi descartado, e o sedimento ressuspenso em 5,0 mL de água mineral natural estéril. Duzentos microlitros dessa suspensão foram inoculados, em triplicata, nas microplacas. A cepa de $P$. aeruginosa ATCC 27853 foi utilizada como controle positivo (CP). Três orifícios foram inoculados com $200 \mu \mathrm{L}$ de caldo BHI sem crescimento e com a água mineral natural estéril, sendo utilizados como controles negativos $(\mathrm{CN})$. Duas microplacas foram incubadas a $25 \pm 2^{\circ} \mathrm{C}$ e duas a $35 \pm 2^{\circ} \mathrm{C}$. Após 24 e 48 -h foi realizada a leitura de uma microplaca incubada em cada temperatura. Cada orifício foi lavado por cinco vezes com água destilada e placa foi mantida a temperatura ambiente durante $45 \mathrm{~min}$. A seguir foi adicionada $200 \mu \mathrm{L}$ de uma solução de cristal violeta $0,41 \%$ (Merck, Alemanha) preparado em solução de etanol a $20 \%$ (v/v) a e a placa foi mantida a temperatura ambiente por $45 \mathrm{~min}$. O conteúdo foi novamente lavado com água destilada por cinco vezes e mantida a temperatura ambiente por $45 \mathrm{~min}$. Em seguida foram adicionados $200 \mu \mathrm{L}$ de etanol a 96\% (Merck, Alemanha) e as microplacas foram mantidas sob agitação de $500 \mathrm{rpm} / 10 \mathrm{~min}$ (IKA, MS3D, EUA). Após, $150 \mu \mathrm{L}$ de cada orifício foram transferidos para uma nova microplaca e foi determinada a densidade ótica (DO) em espectrofotômetro (Biomérieux, Reader 270, França) em comprimento de onda de $600 \mathrm{~nm}$. A interpretação dos resultados foi realizada pela média das nove leituras de cada cultura. As cepas foram classificadas como: não aderentes (NA), fracamente aderentes (FA), moderadamente aderentes (MA) e fortemente aderentes (FMA), segundo o critério descrito por Stepanovic et al. (2007):

$$
\mathrm{NA} \rightarrow \mathrm{DO} \leq \mathrm{DO} \text { do } \mathrm{CN}\left(\mathrm{DO}_{\mathrm{C}}\right) ; \mathrm{FA} \rightarrow \mathrm{DO}_{\mathrm{C}}<\mathrm{DO}<2 \mathrm{x} \mathrm{DO}_{\mathrm{C}} ; \mathrm{MA} \rightarrow 2 \mathrm{x} \mathrm{DO}_{\mathrm{C}}<\mathrm{DO}<
$$
$4 \times \mathrm{DO}_{\mathrm{C}} ; \mathrm{FMA} \rightarrow 4 \times \mathrm{DO}_{\mathrm{C}}<\mathrm{DO}$.

\subsection{Avaliação da susceptibilidade a antimicrobianos}

A avaliação da suscetibilidade dos isolados de $P$. aeruginosa frente aos antimicrobianos foi realizada pelo teste de difusão em ágar (Kirbi-Bauer) baseada nos critérios do CLSI (2012). Foram utilizados discos (produzidos por Diagnósticos Microbiológicos Especializados, Brasil) de doze antibióticos: Imipenem (IPM; $10 \mu g$ ), Ceftazidima (CAZ; $30 \mu g$ ), Meropenem (MER; $10 \mu g$ ), Piperacilina/Tazobactam (PIT; 100/10 $\mu g$ ), Cefepima (CPM; $30 \mu g$ ), Levofloxacina (LVX; $5 \mu g$ ), Tobramicina (TOB; $10 \mu g$ ), Amicacina (AMI; $30 \mu g$ ), Aztreonam (ATM; $30 \mu g$ ), Gentamicina (GEN; $10 \mu g$ ), Ticarcilina/Ácido clavulânico (TAC; 75/10 $\mu g$ ) e Ciprofloxacina (CIP; $5 \mu g$ ).

\section{RESULTADOS E DISCUSSÃO}

As amostras que apresentaram contaminação por algum grupo de micro-organismos descrito na RDC n. ${ }^{\circ}$ 275/05 estão descritas na Tabela 1. Das 80 amostras analisadas, 40 $(50,0 \%)$ apresentaram qualidade microbiológica insatisfatória segundo os critérios da 
resolução supracitada. Quatro amostras (5,0\%) apresentaram CSR, nove $(11,2 \%)$ apresentaram coliformes totais e uma $(1,2 \%)$ apresentou coliformes termotolerantes. $\mathrm{O}$ maior índice de amostras insatisfatórias foi devido à presença de $P$. aeruginosa, que foi detectada em 38 amostras $(47,5 \%)$. Nenhuma amostra apresentou contaminação por enterococos.

Tabela 1. Caracterização microbiológica das amostras de água mineral natural que apresentaram qualidade insatisfatória segundo os parâmetros estabelecidos pela RDC nº 275/05 (Brasil, 2005).

\begin{tabular}{|c|c|c|c|c|}
\hline Amostra & $\begin{array}{c}\text { Coliformes } \\
\text { termotolerantes }\end{array}$ & $\begin{array}{l}\text { Coliformes } \\
\text { totais }\end{array}$ & $\begin{array}{c}\text { Clostrideos sulfito } \\
\text { redutores }\end{array}$ & P. aeruginosa \\
\hline A1 & Ausência & Ausência & Ausência & $>23$ \\
\hline B1 & Ausência & Ausência & Ausência & 2,2 \\
\hline E5 & Ausência & Ausência & Ausência & $>23$ \\
\hline $\mathrm{F} 2$ & Ausência & Ausência & Ausência & 16 \\
\hline G2 & Ausência & Ausência & Ausência & $>23$ \\
\hline $\mathrm{H} 1$ & Ausência & Ausência & Ausência & 1,1 \\
\hline $\mathrm{J} 1$ & Ausência & Ausência & Ausência & 1,1 \\
\hline $\mathrm{J} 2$ & Ausência & Ausência & Ausência & 5,1 \\
\hline $\mathrm{K} 2$ & Ausência & Ausência & Ausência & $>23$ \\
\hline L1 & Ausência & Ausência & Ausência & 9,2 \\
\hline M1 & Ausência & Ausência & Ausência & 1,1 \\
\hline M2 & Ausência & Ausência & Ausência & 1,1 \\
\hline M3 & Ausência & Presença & Presença & 1,1 \\
\hline M4 & Presença & Presença & Presença & 6,9 \\
\hline Q2 & Ausência & Ausência & Ausência & $>23$ \\
\hline $\mathrm{S} 1$ & Ausência & Presença & Ausência & 2,2 \\
\hline U1 & Ausência & Ausência & Ausência & 2,2 \\
\hline U3 & Ausência & Presença & Ausência & 2,2 \\
\hline U5 & Ausência & Ausência & Ausência & 3,6 \\
\hline U6 & Ausência & Ausência & Ausência & 1,1 \\
\hline U7 & Ausência & Ausência & Presença & $<1,1$ \\
\hline U8 & Ausência & Ausência & Ausência & 5,1 \\
\hline U9 & Ausência & Ausência & Ausência & 9,2 \\
\hline U10 & Ausência & Presença & Ausência & 12 \\
\hline U11 & Ausência & Presença & Ausência & 6,9 \\
\hline U12 & Ausência & Presença & Ausência & 6,9 \\
\hline U13 & Ausência & Ausência & Ausência & 2,2 \\
\hline U15 & Ausência & Ausência & Ausência & 1,1 \\
\hline U16 & Ausência & Ausência & Presença & $>23$ \\
\hline U17 & Ausência & Ausência & Ausência & $>23$ \\
\hline Y1 & Ausência & Ausência & Ausência & 1,1 \\
\hline $\mathrm{Z1}$ & Ausência & Ausência & Ausência & 16 \\
\hline $\mathrm{Z4}$ & Ausência & Ausência & Ausência & 1,1 \\
\hline A'1 & Ausência & Ausência & Ausência & 5,1 \\
\hline$C^{\prime} 2$ & Ausência & Ausência & Ausência & 1,1 \\
\hline D'1 & Ausência & Presença & Ausência & $<1,1$ \\
\hline D'2 & Ausência & Presença & Ausência & 23 \\
\hline G'1 & Ausência & Ausência & Ausência & 9,2 \\
\hline G'2 & Ausência & Ausência & Ausência & $>23$ \\
\hline G'3 & Ausência & Ausência & Ausência & 2,2 \\
\hline
\end{tabular}

Nota: Coliformes termotolerantes, coliformes totais e clostrídeos sulfito redutores - Ausência ou presença em $100 \mathrm{~mL}$; . aeruginosa - Número mais provável/100 mL. 
O índice de amostras insatisfatórios obtidos neste estudo foi semelhante ao relatado por outros autores, que ao analisarem águas minerais naturais comercializados em garrafões de $20 \mathrm{~L}$ encontraram um percentual de amostras insatisfatórias variando de 23,8-71,0\% (Sant'ana et al., 2003; Farache Filho e Dias, 2008; Brandao et al.; 2012). Das 33 marcas analisadas neste estudo, apenas 14 marcas $(42,4 \%)$ apresentaram qualidade microbiológica satisfatória para todas as amostras analisadas. Este resultado foi distinto do relatado por Brandao et al. (2012) que ao analisarem a qualidade 15 marcas, observaram que nenhuma marca apresentou qualidade satisfatória em todas as amostras analisadas.

$P$. aeruginosa foi o micro-organismo que representou maior prevalência, com uma concentração variando de 1,1 a $>23 \mathrm{NMP} / 100 \mathrm{~mL}$ (Tabela 1). Trinta amostras $(78,9 \%)$ apresentaram concentração entre 1,1 a $23 \mathrm{NMP} / 100 \mathrm{~mL}$, enquanto que as demais apresentaram o resultado $>23 \mathrm{NMP} / 100 \mathrm{~mL}$. Esse resultado foi similar ao obtido por Brandao et al. (2012), que observaram que 78,6\% das amostras contaminadas por $P$. aeruginosa apresentavam-se na mesma faixa de concentração. Outros autores relataram um maior percentual de amostras de águas minerais naturais em garrafões de $20 \mathrm{~L}$ contaminadas por $P$. aeruginosa, com prevalência de 50,0\% (Nascimento et al., 2000) e 67,7\% (Brandao et al, 2012). A menor prevalência de $P$. aeruginosa observada neste estudo, cujo resultado foi de 38 amostras insatisfatórias para este micro-organismo (47,5\%), pode estar associada ao fato de que as amostras foram coletadas após a publicação da RDC n. ${ }^{\circ}$ 173/2006 (Brasil, 2006), que regulamentou as BPF para os produtores de água mineral natural. Além disso, em 2009 foi publicado a Portaria $n^{\circ}$ 358/2009 (Brasil, 2009) que estipulou um prazo de validade de três anos para as embalagens plástico-garrafão de 20 L. Contudo, o índice de $50 \%$ de amostras insatisfatórias obtido neste estudo indica que ainda estão ocorrendo falhas por parte dos produtores, que devem cumprir a norma de BPF assim como o setor regulador quanto a sua fiscalização desse cumprimento. Além disso, o prazo de três anos talvez ainda não seja suficiente para garantir a integridade das embalagens reutilizáveis de $20 \mathrm{~L}$.

Os resultados da produção de biofilme pelas cepas de $P$. aeruginosa e do perfil de susceptibilidade a antimicrobianos estão apresentados na Tabela 2. Não foi possível isolar cepas de $P$. aeruginosa das amostras $\mathrm{J} 2, \mathrm{M} 1, \mathrm{U} 8, \mathrm{U} 12, \mathrm{U} 15, \mathrm{Y} 1$ e Z1 pois não houve crescimento de colônias no ágar cetrimide. Isto pode ter ocorrido devido ao tempo maior que o caldo acetamida ficou estocado antes da realização da semeadura no ágar cetrimide, que quando não pode ser realizado em tempo hábil, acabou levando a perda da viabilidade da cultura no caldo.

Outros autores que pesquisaram $P$. aeruginosa em amostras de águas minerais naturais em outros tipos de embalagem não retornáveis, como copos plásticos ou garrafas de politereftalato de etileno, não detectaram amostras contaminadas (Coelho et al., 2007; Bernardo, 2009; Neta et al., 2013). Isto indica que as águas minerais comercializadas em garrafões reutilizáveis de 20 L estão mais sujeitas à contaminação. Esse fato está relacionado principalmente a ineficiência das etapas de limpeza e desinfecção dos garrafões, uma vez que o biofilme formado nestas embalagens não é facilmente removível durante estas etapas (Farache Filho e Dias, 2008).

Neste estudo, a pesquisa de formação de biofilme foi testada nas temperaturas de $25 \pm 2{ }^{\circ} \mathrm{C}$, que simula a temperatura ambiente, onde as embalagens de $20 \mathrm{~L}$ são normalmente estocadas no comércio, e $35 \pm 2{ }^{\circ} \mathrm{C}$, que é próxima da temperatura ótima de crescimento de $P$. aeruginosa (Koneman et al., 2001) e comum em dias de calor no Estado do Rio de Janeiro.

Avaliou-se a produção em dois tipos de matrizes, uma em caldo BHI, que é um meio de cultivo rico em nutrientes, e outra em água mineral natural estéril, que apresenta pequena quantidade de nutrientes, de forma a comparar a produção do biofilme na própria matriz em que os micro-organismos se encontram. 
Tabela 2. Produção de biofilme e perfil de susceptibilidade à antimicrobianos das cepas de $P$. aeruginosa

\begin{tabular}{|c|c|c|c|c|c|c|c|c|c|c|}
\hline \multirow{4}{*}{ Amostra } & \multicolumn{8}{|c|}{ Produção de biofilme } & \multirow{2}{*}{\multicolumn{2}{|c|}{ Antibiograma }} \\
\hline & \multicolumn{4}{|c|}{$\begin{array}{c}\text { Caldo infusão cérebro } \\
\text { coração }\end{array}$} & \multicolumn{4}{|c|}{ Água mineral natural estéril } & & \\
\hline & \multicolumn{2}{|c|}{$25^{\circ} \mathrm{C}$} & \multicolumn{2}{|c|}{$35^{\circ} \mathrm{C}$} & \multicolumn{2}{|c|}{$25^{\circ} \mathrm{C}$} & \multicolumn{2}{|c|}{$35^{\circ} \mathrm{C}$} & \multirow[b]{2}{*}{ Intermediário } & \multirow[b]{2}{*}{ Resistente } \\
\hline & 24-h & 48-h & 24-h & 48-h & 24-h & 48-h & 24-h & 48-h & & \\
\hline A1 & FA & FA & NA & FA & FA & FA & FA & FA & $*$ & $*$ \\
\hline B1 & FTA & FTA & MA & MA & FA & FA & FA & FA & $*$ & $*$ \\
\hline E5 & MA & FTA & FA & MA & FA & FA & FA & FA & $*$ & $*$ \\
\hline $\mathrm{F} 2$ & FA & FA & NA & FA & MA & FA & FA & FA & TAC & CAZ, CPM, ATM \\
\hline $\mathrm{G} 2$ & MA & MA & MA & FA & FA & FA & FA & FA & $*$ & $*$ \\
\hline $\mathrm{H} 1$ & MA & MA & MA & MA & FTA & FTA & MA & FA & ATM, TAC, CIP & $*$ \\
\hline $\mathrm{J} 1$ & NA & FA & FA & FA & FA & FA & NA & FA & $*$ & $*$ \\
\hline $\mathrm{K} 2$ & FTA & FTA & MA & MA & FA & FA & FA & FA & $*$ & $*$ \\
\hline L1 & FA & FA & FA & FA & FA & FA & FA & FA & ATM & TAC \\
\hline M2 & FA & FA & NA & FA & FA & FA & FA & FA & ATM, CIP & $\begin{array}{c}\text { TOB, AMI, GEN, } \\
\text { TAC }\end{array}$ \\
\hline M3 & FA & FA & NA & FA & FA & FA & FA & FA & TAC & $*$ \\
\hline M4 & FA & FA & NA & FA & FA & FA & FA & FA & TAC & $*$ \\
\hline Q2 & MA & MA & MA & MA & FA & FA & FA & FA & $*$ & $*$ \\
\hline S1 & FTA & FTA & FTA & FTA & FA & FA & MA & MA & $*$ & $*$ \\
\hline U1 & FA & FA & NA & FA & FA & FA & FA & FA & ATM, TAC & $*$ \\
\hline U3 & FA & FA & FA & MA & FA & FA & FA & FA & $*$ & $*$ \\
\hline U5 & FA & FA & FA & FA & FA & FA & FA & FA & TAC & $*$ \\
\hline U6 & FA & FA & FA & FA & FA & FA & FA & FA & $*$ & $*$ \\
\hline U9 & FA & FA & FA & FA & FA & FA & FA & FA & $*$ & * \\
\hline U10 & FA & FA & FA & MA & FA & MA & FA & FA & ATM, TAC & $*$ \\
\hline U11 & FTA & MA & MA & FA & MA & MA & FA & MA & ATM, TAC & $*$ \\
\hline U13 & FA & FA & FA & FA & FA & FA & FA & FA & ATM & TAC \\
\hline U16 & FA & FA & NA & FA & FA & FA & MA & FA & ATM, TAC & $*$ \\
\hline U17 & FA & FA & FA & FA & FA & FA & FA & FA & TAC & $*$ \\
\hline $\mathrm{Z4}$ & FA & FA & FA & FA & FA & FA & FA & FA & ATM & TAC \\
\hline A'1 & FA & FA & MA & FA & FTA & FTA & FA & FA & ATM, TAC & $*$ \\
\hline$C^{\prime} 2$ & FA & FA & FA & FA & MA & FTA & MA & MA & TAC & $*$ \\
\hline D'2 & MA & FTA & MA & FTA & FA & FA & FA & FA & $*$ & $*$ \\
\hline G'1 & FA & FA & FA & FA & FA & FA & FA & FA & $*$ & ATM, TAC \\
\hline G'2 & FA & FA & FA & FA & FA & FA & FA & FA & $*$ & $*$ \\
\hline $\mathrm{G}^{\prime} 3$ & FA & FA & FA & FA & FA & FA & FA & FA & $*$ & TAC \\
\hline $\begin{array}{c}P . \\
\text { aeruginosa } \\
\text { ATCC27853 }\end{array}$ & FTA & FTA & FTA & FTA & FTA & FTA & MA & MA & NR & NR \\
\hline
\end{tabular}

Nota: FA- Fracamente aderente; NA- Não aderente; *- Sensível a todos os antimicrobianos testados; FTA- Fortemente aderente; MA - Moderadamente aderente; TAC- Ticarcilina/Ácido clavulânico; CAZ - Ceftazidima, COM - Cefepima; ATM- Aztreaonam; CIP - Ciprofloxacina; TOB - Tobramicina; AMI - Amicacina; GEN- Gentamicina; NR - Não realizado.

A maioria das cepas de $P$. aeruginosa (A1, J1, L1, M2, M3, M4, U1, U5, U6, U9, U13, U17, Z4, G'1, G'2 e G'3) foram classificados como NA ou FA, tanto no caldo BHI quanto na água mineral estéril (Tabela 2). Considerando como resultado positivo para produção de biofilme a classificação de MA ou FTA em pelo menos uma das temperaturas estudadas (25 ${ }^{\circ} \mathrm{C}$ ou $35^{\circ} \mathrm{C}$ ) e durante um dos períodos de incubação (24 ou 48-h); as cepas B1, E5, G2, K2, Q2, U3 e D'2 foram capazes de produzir biofilme no BHI, mas não foram capazes de produzir biofilme na água mineral. Provavelmente, estas cepas necessitam de uma quantidade de nutrientes para produção do EPS e/ou dos demais componentes do biofilme que só estavam disponíveis no BHI. Por outro lado, as cepas H1, S1, U10, U11 e A'1 produziram biofilme tanto no BHI quanto na água mineral, indicando que a concentração de nutrientes no meio não foi o fator limitante para produção do biofilme. Já as cepas F2, U16 e C'2 produziram 
biofilme apenas na água mineral. Neste caso, a escassez de nutrientes pode ter sido a responsável pela ativação de genes para a produção de biofilme. A cepa de $P$. aeruginosa ATCC 27853 utilizada como CP apresentou produção de biofilme em todos os experimentos.

Muitos estudos apontam que o aumento de bis-(3',5')-di-guanosina monofosfato cíclico (c-diGMP) intracelular atua como um segundo mensageiro que leva a expressão de fímbrias em $P$. aeruginosa que auxiliam no formação do biofilme (Häußler, 2008). Determinados fatores ambientais, como luz, oxigênio e nutrientes, podem modular a expressão de c-diGMP (Kulasakara et al., 2006). De acordo com Yasuhiko et al. (2012), o biofilme formado devido ao aumento de c-diGMP intracelular tende a ser mais aderente e robusto do que em cepas que produzem biofilme sem ativar esta via. Logo, as cepas que foram classificadas como FTA (B1, E5, H1, K2, S1, U11, A'1, C'2 e D'2) provavelmente utilizaram a via c-diGMP para produção de biofilme. A identificação de cepas de $P$. aeruginosa fortemente produtoras de biofilme já foi descrito por Bernardo (2009) em ensaios que avaliaram a produção de biofilme em BHI a $37^{\circ} \mathrm{C}$ durante 24-h.

O grau de formação do biofilme produzido foi considerado com maior adesão quando incubado à temperatura de $25^{\circ} \mathrm{C}$ (Tabela 2). Contudo, as cepas S1, U3 e U16 não apresentaram este perfil, produzindo biofilme apenas na temperatura de $35^{\circ} \mathrm{C}$. Segundo Mohamed e Huang (2007), a temperatura tem um efeito na produção de EPS, afetando de forma direta a capacidade de adesão e formação de biofilme, bem como a forma com que as bactérias neste estado lidam com os estresses ambientais. A maior formação do biofilme na temperatura de $25^{\circ} \mathrm{C}$ demostra que quando as águas estão contaminadas por $P$. aeruginosa $\mathrm{o}$ biofilme pode ser formado mesmo na temperatura de prateleira (Bernardo, 2009).

Os isolados de $P$. aeruginosa apresentaram sensibilidade a maioria dos antibióticos, sendo 13 isolados $(34,2 \%)$ sensíveis a todos os antibióticos. Esse resultado foi similar ao relatado por Bernardo (2009), que avaliou o perfil de susceptibilidade de isolados de $P$. aeruginosa de amostras de água mineral e observaram que as cepas foram sensíveis a todos os antibióticos. Contudo, 12 cepas $(38,7 \%)$ apresentaram resistência intermediária a TAC, 10 $(32,2 \%)$ a ATM e dois $(6,4 \%)$ a CIP (Tabela 2$)$. Seis cepas $(19,4 \%)$ apresentaram resistência a TAC e duas $(6,4 \%)$ a ATM (Tabela 2 ). A cepa F2 apresentou resistência a CAZ e CPM; e a cepa M2 apresentou resistência a TOB, AMI e GEN (Tabela 2), sendo os isolados que apresentaram maior resistência aos antimicrobianos. Resultado semelhante foi relatado por Vaz-Moreira et al. (2012) que ao analisarem diferentes tipos de água, incluindo água mineral natural, observaram que as cepas de $P$. aeruginosa possuíam maior resistência a TAC (80\%), seguidas de CAZ e CPM (18 e 2\%, respectivamente) e não foi observada nenhuma resistência a IPM e MER. Os antibióticos ATM, CAZ, CPM e TAC pertencem ao grupo dos $\beta$ lactâmicos (CLSI, 2012). Resistência a esta classe de antimicrobianos já foi descrita em cepas de $P$. aeruginosa, sendo os mecanismos de resistência mais comuns a difusão deficiente ou porinas alteradas e a produção de $\beta$-lactamase de amplo espectro. Outros mecanismos também descritos são a alteração de proteínas fixadoras de penicilinas e a baixa força motriz de prótons (Konemann et al., 2001). Segundo Dunn e Wunderink (1995) os $\beta$-lactâmicos variam na aptidão em induzir a produção de $\beta$-lactamase em $P$. aeruginosa. Logo, isso também pode explicar o porquê determinadas cepas apresentaram resistência a alguns $\beta$ - lactâmicos e outras não. Os antibióticos TOB, AMI e GEN pertencem ao grupo dos aminoglicosídeos que necessitam cruzar a membrana citoplasmática bacteriana antes de iniciar seus efeitos letais (Beltrame et al., 1999). A resistência a esta classe de antimicrobianos em $P$. aeruginosa provém da modificação do antibiótico mediada por enzimas e/ou difusão deficiente ou porinas alteradas (Koneman et al., 2001).

Comparando a produção de biofilme com o perfil de susceptibilidade a antimicrobianos, nota-se que a maior parte das cepas que apresentou resistência intermediária ou resistência a antimicrobianos foram classificadas como FA (Tabela 2). As cepas F2, H1, U10, U11, U16, 
A'1 e C'2 que produziram biofilme na água mineral apresentaram resistência e/ou resistência intermediária a determinados antimicrobianos. De acordo com Häußler (2008), o aumento dos níveis de c-diGMP também pode aumentar a resistência a antibióticos. Logo, estas cepas podem ter utilizado o mecanismo de aumento c-diGMP intracelular e consequentemente apresentaram maior resistência a antimicrobianos.

\section{CONCLUSÃO}

Concluiu-se que isolados de $P$. aeruginosa foram capazes de produzir biofilme em caldo BHI (22,6\%), em água mineral natural $(9,7 \%)$ e em ambos $(16,1 \%)$. Esta produção foi maior no caldo BHI e na temperatura de $25^{\circ} \mathrm{C}(32,3 \%)$. Algumas cepas apresentaram resistência $(51,6 \%)$ ou resistência intermediária $(22,6 \%)$ a antimicrobianos, o que pode prejudicar o tratamento de pacientes no caso de infecções que tenham como agente estes microorganismos. Metade das amostras analisadas (40 amostras) apresentou qualidade microbiológica insatisfatória segundo a legislação brasileira vigente, principalmente devido à contaminação por $P$. aeruginosa. Desta forma sugerimos que os fabricantes estejam atentos ao cumprimento das BPF, principalmente na etapa de higienização das embalagens retornáveis de $20 \mathrm{~L}$, de forma a garantir a eliminação do biofilme possivelmente presente.

\section{AGRADECIMENTOS}

Agradecemos ao INCQS/Fiocruz pelo financiamento deste estudo e pela concessão de bolsa de iniciação científica (PIBIC-CNPq) à Aline Pedrosa. Agradecemos às Vigilâncias Sanitárias Municipais e Estadual pela coleta das amostras analisadas neste estudo.

\section{REFERÊNCIAS}

AMERICAN PUBLIC HEALTH ASSOCIATION - APHA. Standard methods for the examination of water and wastewater. $22^{\text {st }} \mathrm{Ed}$. Washington, 2012.

BAQUERO, F.; MARTÍNEZ, J. L.; CANTÓN, R. Antibiotics and antibiotic resistance in water environments. Current Opinion in Biotechnology, Inglaterra, v. 19, n.3, p. 260 265, jun., 2008. http://dx.doi.org/10.1016/j.copbio.2008.05.006

BELTRAME, R. E.; SILVA FILHO, C. R.; TANAKA, I. I.; SORNAS, A. M. F. Pseudomonas aeruginosa - antibioticoterapia e perfil de resistência de cepas isoladas de UTIs dos Hospitais da Faculdade de Medicina de Marília. RBM Revista Brasileira de Medicina, São Paulo, v.56, n.11, p. 1132-1144, 1999. Disponível em: $<$ http://www.moreirajr.com.br/revistas.asp?fase=r003\&id_materia=414>. Acesso em maio 2014.

BERNARDO S. P. C. Avaliação da Suscetibilidade a Antimicrobianos e Formação de Biofilmes em Pseudomonas aeruginosa Isoladas de água Mineral. 2009. $70 \mathrm{f}$. Dissertação (Mestrado em Vigilância Sanitária) - Fundação Oswaldo Cruz, Instituto Nacional de Controle de Qualidade em Saúde, Rio de Janeiro, 2009.

BRANDAO, M. L. L.; ROSAS, C. O.; MEEIROS, V. M.; WARNKEN, M. B.; BRICIO, S. M. L.; SILVA, A. M. L. da et al. Comparação das técnicas do Número Mais Provável (NMP) e de filtração em membrana na avaliação da qualidade microbiológica de água mineral natural. Revista do Instituto Adolfo Lutz, São Paulo, v. 71, n. 1, p. 32-39, 2012. 
BRASIL. Ministério da Saúde. Resolução RDC n. ${ }^{\circ} 275$ de 22 de setembro de 2005. Regulamento técnico de características microbiológicas para água mineral natural e água natural. Diário Oficial [da] República Federativa do Brasil, Brasília, DF, v. 184, 23 set. 2005, Seção 1, p. 377.

BRASIL. Ministério da Saúde. Resolução RDC n. ${ }^{\circ} 173$ de 13 de setembro de 2006. Dispõe sobre o regulamento técnico de boas práticas para industrialização e comercialização de água mineral natural e água mineral. Diário Oficial [da] República Federativa do Brasil, Brasília, DF, v. 178, 15 set. 2006, Seção 1, p. 60.

BRASIL. Ministério de Minas e Energia. Departamento Nacional de Produção Mineral. Portaria $\mathrm{n}^{\mathbf{0}} 358$, de 21 de setembro de 2009. Altera a Portaria $\mathrm{n}^{\mathbf{0}} 387$, de 19 de setembro de 2008. Diário Oficial [da] República Federativa do Brasil, Brasília, DF, v. 181, 22 set. 2009, Seção 1, p. 51.

BRASIL. Ministério de Minas e Energia. Departamento Nacional de Produção Mineral. Sumário Mineral Brasileiro 2013. Disponível em:

$<$ https://sistemas.dnpm.gov.br/publicacao/mostra_imagem.asp?IDBancoArquivoArquiv o=8963>. Acesso em: 15 jan. 2014.

BRASIL. Ministério da Saúde. Secretaria de Vigilância em Saúde. Departamento de Vigilância Epidemiológica. Coordenação Geral de Doenças Transmissíveis. Vigilância epidemiológica das doenças transmitidas por alimentos: abril - 2013. Brasília, 2013.

CLINICAL AND LABORATORY STANDARDS INSTITUTE. Performance Standards for antimicrobial susceptibility testing; twenty-second informational supplement. M100-S22, v. 32, n. 3, p. 62 - 63. Wayne, 2012.

COELHO, D. A.; FARIA E SILVA, P. M. de; VEIGA, S. M. O. M. Avaliação da qualidade microbiológica de águas minerais naturais comercializadas em supermercados da cidade de Alfenas, MG. Revista Higiene Alimentar, São Paulo, v.21, n. 151, p. 88-92, maio 2007.

DUNN, M.; WUNDERINK, R. G. Ventilator-associated pneumonia caused by Pseudomonas infection. Clinics in Chest Medicine, USA, v.16, n.1, p. 95-109, 1995.

FARACHE FILHO, A.; DIAS, M. F. F. Qualidade microbiológica de águas minerais em galões de 20 litros. Alimentos e Nutrição, Araraquara, v. 19, n.3, p. 243-248, jul./set. 2008.

HÄUBLER, S. Pseudonomas aeruginosa Biofilms: impact of small colony variants on chronic persistent infections. In: CORNELIS, P. Pseudomonas Genomic and Molecular Biology. Norfolk: Horizon Scientific Press, 2008. p. 159-175.

INTERNATIONAL ORGANIZATION FOR STANDARDIZATION - ISO. ISO 6461 Water Quality - Detection and enumeration of the spores of sulfite-reducing anaerobes (clostridia), Part 1 - Method by enrichment in a liquid medium. $1^{\text {st }}$ Ed. [S.1.], 1986.

KIM, H.; FENG, P. Bottled water. In: DOWNES, F. P.; ITO, K.; AMERICAN PUBLIC HEALTH ASSOCIATION. Compendium of methods of examination of foods. 4 . ed. Washington: APHA, 2001. p. 573-576. 
KONEMAN, W. E.; ALLEN, S. D.; JANDA, W. M.; SCHRECKENBERGER, P. C.; WINN JR., W. C. Bacilos Gram-Negativos não-fermentadores. In: KONEMAN, E. W. Diagnóstico microbiológico - texto e atlas colorido. 5. ed. Rio de Janeiro: Médica e Científica, 2001. p. 263-329.

KULASAKARA, H.; LEE, V.; BRENCIC, A.; LIBEATI, N.; URBACH, J.; MIYATA, S. et al. Analysis of Pseudomonas aeruginosa diguanylate cyclases and phosphodiesterases reveals a role for bis-( $\left.3^{\prime}-5^{\prime}\right)$-cyclic-GMP in virulence. Proceedings of the National Academy of Sciences USA, Washington, v. 103, n. 8, p. 2839-2844, 2006. http://dx.doi.org/10.1073/pnas.0511090103

LECLERC, H.; MOREAU, A. Microbiological safety of natural mineral water. FEMS Microbiology Reviews, Reino Unido, v. 26, n. 2, p. 207-222, 2002. http://dx.doi.org/10.1111/j.1574-6976.2002.tb00611.x

MOHAMED, J. A.; HUANG, D. B. Biofilm formation by enterococci. Journal of medical microbiology, v. 56, p. 1581 - 1588, dec. 2007. http://dx.doi.org/10.1099/jmm.0.473310

NASCIMENTO, A. R.; AZEVEDO, T.K.L.; FILHO, N.E.M.; ROJAS, M. O. A. I. Qualidade microbiológica das águas minerais consumidas na cidade de São Luís - MA. Higiene Alimentar, Campinas, v. 14, n. 76, p. 69-72, 2000.

NETA, M. S.; LEAL, M. P. N; REIS, A. S. Análise físico-química, microbiológica de água mineral produzida no nordeste e comercializada em Teresina - Piauí. Revista Interdisciplinar, Teresina, v. 6, n. 2, p. 33-37, abr./maio/jun. 2013.

PATEL, J.; SHARMA, M. Differences in attachment of Salmonella enterica serovars to cabbage and lettuce leaves. International Journal of Food Microbiology, Holanda, v. 139, p. 41-47, 2010. http://dx.doi.org/10.1016/j.ijfoodmicro.2010.02.005

SANT'ANA, A.; SILVA, S. C. F. L.; FARANI, I. O. J.; AMARAL, C. H. R.; MACEDO, V. F. Qualidade microbiológica de águas minerais. Ciência e Tecnologia de Alimentos, Campinas, v. 23, p. 190 -194, dez., 2003.

STEPANOVIC, S.; VUKOVIC, D.; HOLA, V.; DI BONAVENTURA, G.; DJUKIĆ, S.; ĆIRKOVIĆ, I. et al. Quantification of biofilm in microtiter plates: overview of testing conditions and practical recommendations for assessment of biofilm production by staphylococci. APMS, v. 115, n. 8, p 891-899, Aug. 2007. http://dx.doi.org/10.1111/j.1600-0463.2007.apm_630.x

VAZ-MOREIRA, I.; NUNES, O. C.; MANAIA, C. M. Diversity and antibiotic resistance in Pseudomonas spp. from drinking water. Science of Total Environment, v. 426, p. 366 - 374, June 2012. http://dx.doi.org/10.1016/j.scitotenv.2012.03.046

WINGENDER, J.; FLEMMING, H. C. Biofilms in drinking water and their role as reservatoir for pathogens. International Journal of Hygiene and environmental Health, Alemanha, v. 214, n. 6, p. 417-423, Nov. 2011. http://dx.doi.org/10.1016/j.ijheh.2011.05.009

WORLD HEALTH ORGANIZATION - WHO. Burden of disease and cost-effectiveness estimates. In: Water sanitation health. 2007. Disponível em: http://www.who.int/water_sanitation_health/diseases/burden/en/. Acesso em: 31 jan. 2014. 
YASUHIKO, I.; BORLEE, B. R.; O’CONNOR, J. R.; HILL, P. J., HARWOOD, C. S.; WOZNIAK, D. J. et al. Self-produced exopolysaccharide is a signal that stimulates biofilm formation in Pseudomonas aeruginosa. Proceedings of the National Academy of Sciences USA, v. 109, n. 50, p. 20632-20636, Dec. 2012. 